\title{
Effects of Discontinuation of Urate-Lowering Therapy: A Systematic Review
}

\author{
Virginie Beslon, $M D^{7}$, Perrine Moreau, $M D^{7}$, Annabel Maruani, MD, PhD², Hubert Maisonneuve, $\mathrm{MD}^{3}$, \\ Bruno Giraudeau, $M D, P h D^{4}$, and Jean-Pascal Fournier, $M D, P h D^{7}$ \\ 'Département de médecine générale, Université de Nantes, Nantes, France; ${ }^{2}$ Services de dermatologie, CHRU de Tours, Université François \\ Rabelais, Tours, France; ${ }^{3}$ Unité des Internistes Généralistes et Pédiatres, Faculté de Médecine, Université de Genève, Geneva, Switzerland; ${ }^{4}$ INSERM \\ CIC1415, CHRU de Tours, Tours, France.
}

BACKGROUND: Urate-lowering therapy (ULT) is associated with low rates of adherence, leading to a potential risk of relapse of gouty arthritis, tophi, or urolithiasis. Our main aim was to identify the recurrence of gouty arthritis, tophi, or urolithiasis after discontinuation of ULT. Secondary aims included an assessment of ULT reintroduction rates and factors associated with relapse.

METHODS: We conducted a systematic literature review of clinical studies investigating the effect of discontinuing any ULT (allopurinol, febuxostat, probenecid, sulfinpyrazone, benzbromarone) in adults on long-term therapy. We searched The Cochrane Central Register of Controlled Trials, MEDLINE, EMBASE, Science Citation Index, and ClinicalTrials.gov from inception to March 2016. Conference abstracts of the ACR/ARHP and EULAR annual conferences were hand-searched. Study quality was assessed using the first eight items of the methodological index for non-randomized studies (MINORS) tool. The review protocol is registered with PROSPERO (CRD42016042048).

RESULTS: A total of 4640 articles were identified, eight of which were ultimately included. Most of these studies predated 2000. MINORS scores ranged from 5 to 10 out of a possible 16. Mean follow-up duration after discontinuation ranged from 12 to 96 months. Five studies focused on discontinuation of ULT in gouty arthritis and tophi, two in urolithiasis, and one in asymptomatic hyperuricemia. Relapse rates were high in gout (36-81\%) and lower in urolithiasis (15\%). Relapses occurred 1-4.5 years after ULT discontinuation. In one study, a low serum urate level before and after ULT discontinuation was associated with lower gout recurrence.

DISCUSSION: Relapse of gout is common although delayed after discontinuation of ULT. Short-term prognosis after ULT discontinuation appears favorable if the serum urate level was low before ULT discontinuation. The results of this review are limited by the paucity of existing studies and their low quality. Further comparative studies should consider larger primary care populations and discontinuation of febuxostat.

KEY WORDS: gout; urolithiasis; hyperuricemia; urate-lowering therapy; deprescriptions.

Virginie Beslon and Perrine Moreau contributed equally to this work.

Received August 8, 2017

Revised October 3, 2017

Accepted November 8, 2017

Published online December 4, 2017
J Gen Intern Med 33(3):358-66

DOI: $10.1007 / \mathrm{s} 11606-017-4233-5$

(c) Society of General Internal Medicine 2017

\section{INTRODUCTION}

Discontinuation of urate-lowering therapy (ULT) is an important issue in the general population. Allopurinol and other ULTs have been reported to be associated with low drug adherence rates. ${ }^{1,2}$ Observational studies have consistently found that 46-90\% of patients with gout do not fully adhere to their ULT regimen. ${ }^{3}$ According to two electronic database studies, one in every two patients with gout will discontinue their ULT during the first year of treatment, and up to three in four within the first 2 years. 4

The main reasons identified by patients for non-adherence and discontinuation of ULT relate to uncertainty regarding long-term effectiveness, fear of adverse reactions, and polypharmacy burden. ${ }^{6-8}$ Other reasons include forgetfulness, refill difficulties, financial concerns, difficulty in swallowing large pills, frequent travel, and preference for alternative medicine. ${ }^{6-8}$ Some physicians also report actively discontinuing ULT in the case of adequate lifestyle modification by patients or the development of renal impairment. ${ }^{9}$

Concerns related to discontinuation of lifelong medications include the risk of relapse of the condition initially treated, the time to relapse, and factors associated with relapse. Other areas of interest include the biological consequences of discontinuation, as well as discontinuation strategies. All of these require a comprehensive assessment in the area of ULT discontinuation. To this end, we conducted a systematic literature review of clinical studies addressing the issue of ULT discontinuation. Our main aim was to identify the recurrence of gouty arthritis, tophi, or urolithiasis after discontinuation of ULT. Secondary aims included the assessment of ULT reintroduction rates and factors associated with relapse.

\section{METHODS}

\section{Protocol and Registration}

This systematic review is registered with the PROSPERO international prospective register of systematic reviews (no. 
CRD42016042048). We followed PRISMA guidelines in conducting this review.

\section{Eligibility Criteria}

We included all types of studies (all randomized controlled trials [RCTs], controlled clinical trials [CCTs], and observational studies [case-control, longitudinal studies]), with the exception of case studies, investigating the effect of discontinuation of ULT in adults (aged 18 years and older) undergoing long-term therapy. Trials and studies assessing discontinuation of any ULT were included: xanthine oxidase inhibitors (allopurinol, febuxostat) or uricosuric agents (probenecid, sulfinpyrazone, benzbromarone). Studies assessing switches between ULTs were excluded. Studies were eligible regardless of the indication for ULT (acute gouty arthritis, tophi, urate nephropathy, uric acid urolithiasis, prophylaxis of chemotherapy-induced hyperuricemia, asymptomatic hyperuricemia, other). Studies assessing only shortterm effects (less than 3 months) of ULT discontinuation were excluded. If the same participants were assessed at different time points or in multiple studies, we extracted and analyzed the data for all follow-up periods, and those with the longest follow-up period were selected for analysis.

The search was limited by language (English and French), and only studies with human subjects were included.

\section{Search Strategy}

The search strategy for MEDLINE was developed in collaboration with two librarians and adapted for each database (Table 1). The following databases were searched from inception to March 2016: the Cochrane Central Register of Controlled Trials (CENTRAL), MEDLINE, EMBASE, Science Citation Index (Web of Science), and ClinicalTrials.gov. Reference lists of articles were inspected for relevant supplemental studies. Conference abstracts of the American College of Rheumatology/Association of Rheumatology Health Professionals (ACR/ARHP) annual meetings (2006-2015) and European League Against Rheumatism (EULAR) annual congresses (2002-2015) were also searched manually.

\section{Study Selection}

Three authors (VB, PM, JPF) independently screened titles and abstracts for inclusion of all potential studies. Any discrepancies were discussed and resolved by consensus. Full copies were obtained for all studies deemed potentially relevant. The same three reviewers (VB, PM, JPF) independently assessed these studies for inclusion; any disagreements were resolved by consensus. Duplicates were identified and excluded, and multiple reports of the same study were collated so that each study, rather than each report, was the unit of interest in the review.

\section{Data Collection Process}

Data were extracted independently by two authors (VB and PM); discrepancies were identified and resolved by consulting
Table 1 Defined Search Strategy for the Extraction of Pertinent Studies from MEDLINE

\begin{tabular}{ll}
\hline \hline Outcome & Descriptor \\
\hline Search for urate-lowering drug & 1. Allopurinol \\
& 2. Febuxostat \\
3. Probenecid \\
4. Benzbromarone \\
5. Urate lowering \\
6. Xanthine oxidase inhibitor \\
7. Antihyperuricemic \\
8. Anti-hyperuricemic \\
9. Gout suppressants \\
10. OR/1-9 \\
11. Stop* \\
12. Withdraw* \\
13. Cess* \\
14. Discontinu* \\
15. Withhold* \\
16. Step-down \\
17. Deprescri* \\
18. OR/11-17 \\
19. Animals (MeSH) \\
Search for human studies & 20. Not humans (MeSH) \\
Search for combinations & 22. NOT 19-21 \\
&
\end{tabular}

another author (JPF). A standardized, pre-piloted form was used to extract data from the included studies for assessment of study quality and evidence synthesis.

\section{Data ltems}

The following information was extracted: (1) methods (study design, total duration of study, number of study centers and location, study settings, mean follow-up after ULT discontinuation, date of study); (2) participant characteristics (number, mean age, age range, sex, disease duration, severity of condition, diagnostic criteria, baseline data, inclusion criteria, and exclusion criteria); (3) interventions (type of discontinuation: abrupt or tapering), comparison (if applicable), concomitant medications, excluded medications; (4) outcomes (recurrence of gouty arthritis, tophi, urate nephropathy or urolithiasis, ULT reintroduction, serum urate level, serum creatinine level, time of measurement); (5) notes (funding, and notable declarations of interest by authors).

\section{Quality of Individual Studies}

Two review authors (VB and PM) independently assessed the quality of included studies using the Methodological Index for Non-Randomized Studies (MINORS) tool. ${ }^{10}$ Any disagreements were resolved by discussion or by involving another author (JPF). The MINORS tool identifies 12 items, including 8 specifically designed for non-comparison studies: a clearly stated aim, the inclusion of consecutive patients, a prospective collection of data, endpoints appropriate to the aim of the study, an unbiased assessment of the study endpoints, a follow-up period appropriate to the aim of the study, loss to follow-up less than 5\%, a prospective calculation of the study size, an adequate control group, contemporary groups, baseline equivalence of groups, and adequate statistical analysis. 
The items are scored as follows: $0=$ reported, $1=$ reported but inadequate, 2 = reported and adequate. The highest score possible was 16 for non-comparative studies and 24 for comparative studies.

\section{Data Synthesis}

After extracting data from each study, structured tables were used to summarize the main characteristics of included studies, and a narrative synthesis of the other findings was provided. It was anticipated that there would be limited scope for metaanalysis because of the range of different outcomes measured across the small number of existing studies. A forest plot of relapse rates is included for illustrative purposes.

\section{RESULTS}

\section{Study Selection}

A total of 4640 citations were retrieved (Fig. 1). Suppression of duplicate publications and screening of titles and abstracts yielded 21 articles for full-paper review. Among these, 13 were excluded, and the remaining eight studies were included in this review.

\section{Study Characteristics}

The design, methods, number of patients enrolled, and other characteristics of included studies are presented in Table 2. All studies were observational. No ongoing studies were reported from ClinicalTrials.gov. Most of the studies predated 2000 and were small, ranging from 10 to 211 subjects. Most of the patients were male, with mean age ranging from 41.8 to 59.9 years. All studies were conducted within a single country and in hospital settings.

Five studies focused on ULT in gout (gouty arthritis or tophi), two studies in urolithiasis, and one in asymptomatic hyperuricemia.

The mean duration of ULT treatment prior to discontinuation ranged from 17 to 86.4 months. Most (50$100 \%$ ) of the patients were treated with allopurinol. Seven studies assessed the consequences of an abrupt discontinuation of ULT. A tapering strategy was used in only one study, though strategy details were not reported. ${ }^{11}$ Criteria for ULT discontinuation were reported in only two studies. In the first of these, ULT was discontinued because of a side effect in $15 \%$ of patients, and was stopped after full discussion in the remaining cases. ${ }^{12}$ In the second study, all patients had chosen to discontinue ULT. ${ }^{13}$ The mean duration of follow-up after discontinuation ranged from 12 to 96 months. Two studies appeared to use data from overlapping groups of patients $^{13,}{ }^{14}$; the authors of these two studies were contacted and confirmed this possibility. However, they were unable to determine the percentage of patients that may have been included in both studies.

\section{Quality of Studies}

The results of the quality assessment using the MINORS tool are reported in Table 2. MINORS scores ranged from 5 to 10 out of a possible 16. Most studies received scores of 0 for inclusion of consecutive patients, prospective collection of data, and calculation of the study size, due to insufficient clarity and/or quality of study reports.

\section{Clinical and Biological Effects of Discontinuation in Gouty Arthritis and Tophi}

Four studies included patients with or without tophus, ${ }^{11}$, 13-15 and one study restricted inclusion to patients with gouty arthritis with normal renal function who were not over-producers of uric acid. ${ }^{12}$ Two studies ${ }^{13,}{ }^{14}$ used the 1977 American Rheumatism Association (ARA) preliminary criteria for primary gout. ${ }^{16}$ Darmawan et al. used these criteria only if diagnosis by identification of monosodium urate crystals in the synovial fluid was not possible. ${ }^{11}$ Perez-Ruiz et al. defined gouty arthritis based on the identification of monosodium urate crystals from a tophus or synovial fluid aspiration. ${ }^{15}$ In one study, the criteria for gout were not reported. ${ }^{12}$

Gout Relapse and ULT Discontinuation Rates. Relapse rates for gout are presented in Table 3 and Figure 2. Among the five studies included, recurrence of arthritis was reported in 36.4$81 \%$ of patients. The mean time to relapse ranged from 15.8 to 56 months. In the largest study, cumulative relapse rates at 12 , 24,36 , and 48 months were $6.6 \%, 11.4 \%, 20.4 \%$, and $29.4 \%$, respectively. ${ }^{15}$ The earliest relapses occurred 4 months after ULT discontinuation. ${ }^{13}$ Conversely, the longest gout-free follow-up period observed after ULT discontinuation was 96 months. ${ }^{11}$ Overall, final discontinuation rates for ULT were $40.8 \%, 61 \%$, and $80 \%$ in the three studies with contributing data. $11,12,14$

Factors Associated with Gout Relapse. Three studies investigated the association between several factors and gout relapse after ULT discontinuation. Loebl et al. reported that patients with recurrence of gout tended to have higher serum urate levels before discontinuation, though no significant difference was observed (6.2 \pm $0.4 \mathrm{mg} / \mathrm{dl}$ and $5.1 \pm 0.9 \mathrm{mg} / \mathrm{dl}$, respectively). ${ }^{12}$ Gast et al. found that higher BMI, earlier age at onset of gout, higher serum urate level before discontinuation, and longer duration of ULT before discontinuation tended to be associated with higher rates of gout recurrence (no statistically significant association). ${ }^{14}$ In a study by PerezRuiz et al., the only variables independently associated with gout recurrence were a higher serum urate level during ULT treatment (Cox multivariate survival analysis, adjusted HR: 1.57; 95\% CI: 1.18-2.08) and during follow-up after ULT discontinuation (HR: 2.29; 95\% CI: 1.91-2.74). ${ }^{15}$ 


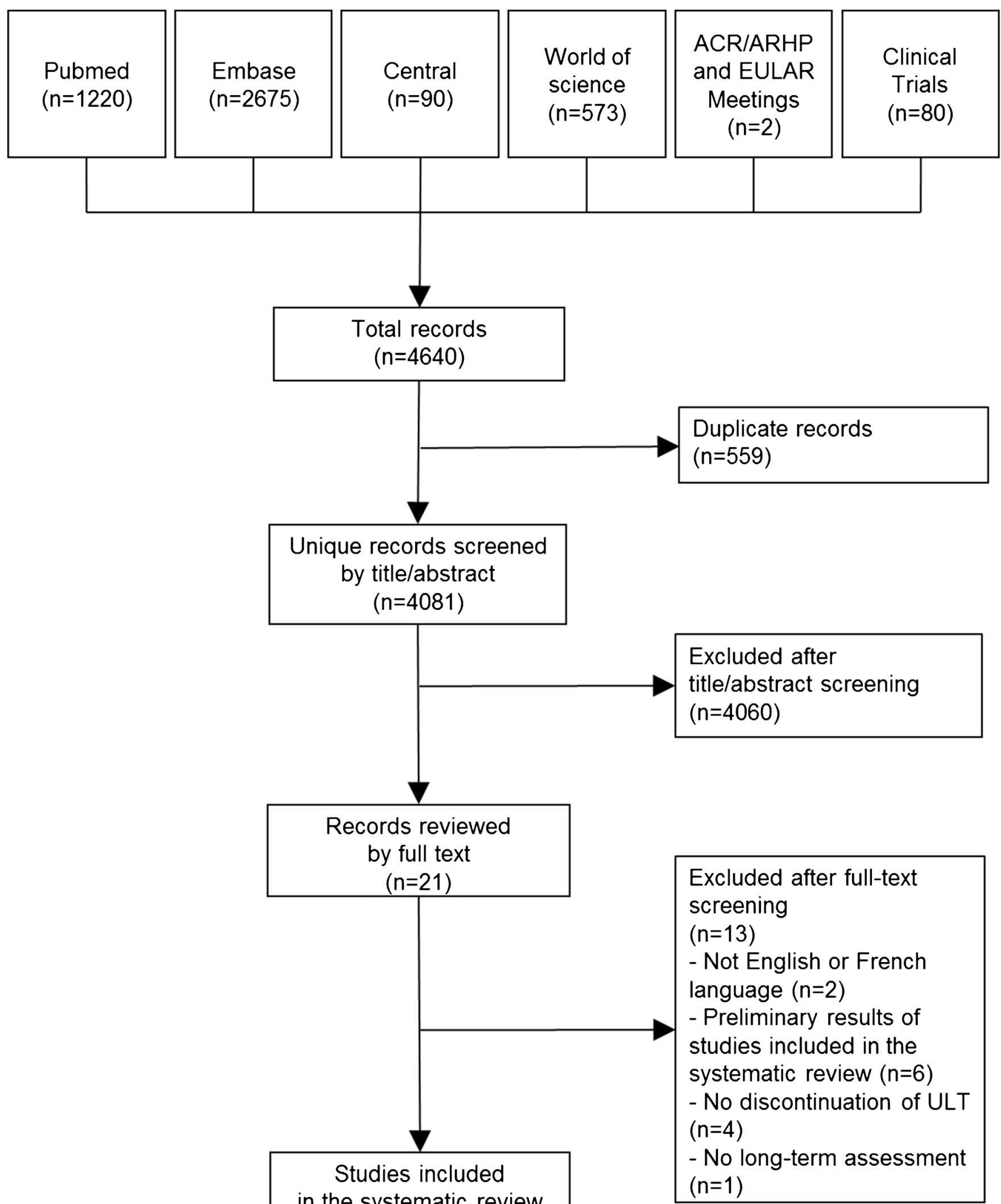

in the systematic review

$$
(n=8)
$$

Figure 1 Flowchart of study selection. 


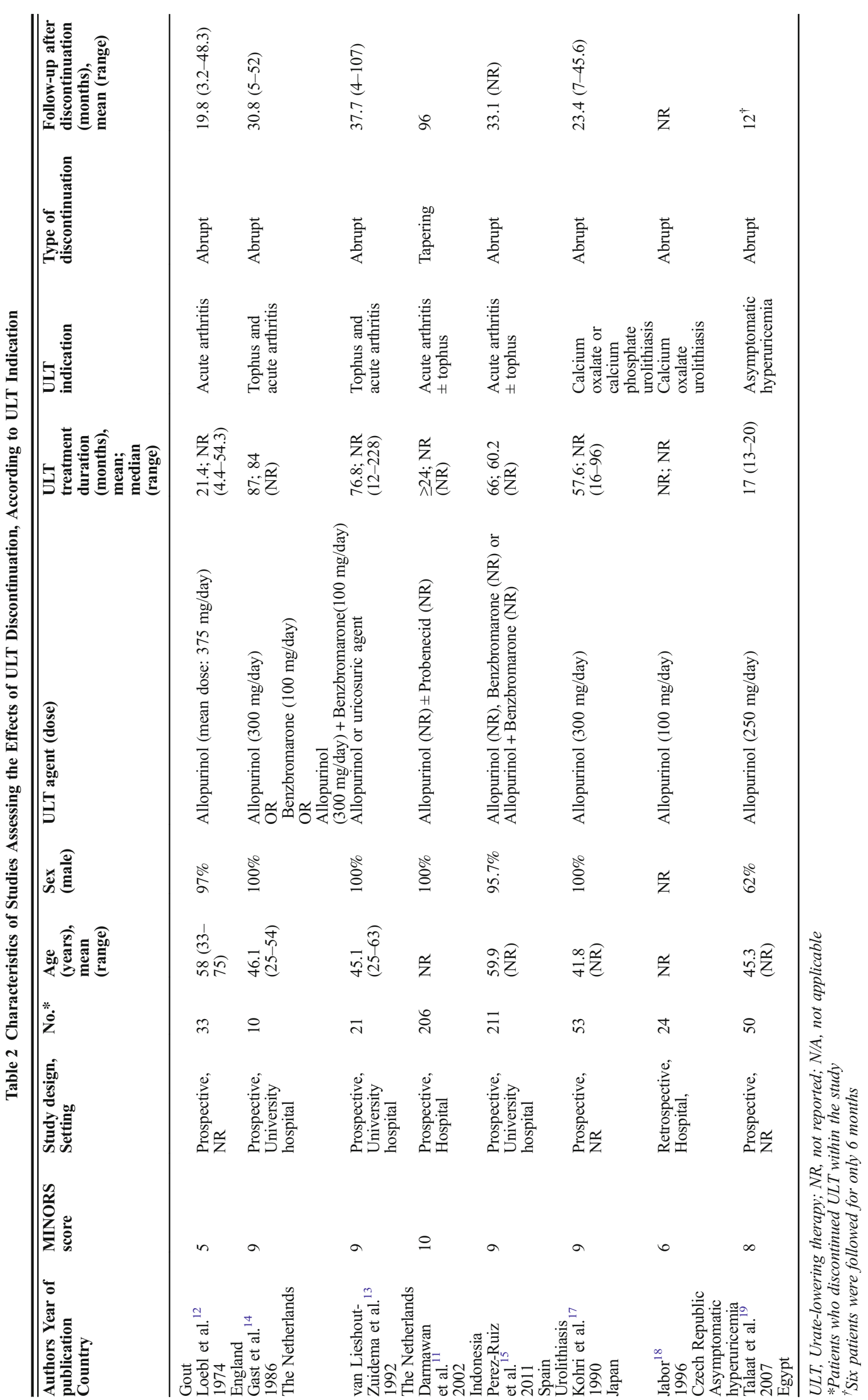


Table 3 Outcomes of Studies Assessing the Effects of ULT Discontinuation, According to ULT Indication

\begin{tabular}{|c|c|c|c|c|c|c|}
\hline \multirow{2}{*}{$\begin{array}{l}\text { Authors Year } \\
\text { of publication } \\
\text { Country }\end{array}$} & \multicolumn{3}{|c|}{ Serum urate level $(\mathrm{mg} / \mathrm{dl})$, mean } & \multirow{2}{*}{$\begin{array}{l}\text { Clinical effects } \\
\text { (relapse) }\end{array}$} & \multirow{2}{*}{$\begin{array}{l}\text { Time to relapse } \\
\text { (months), } \\
\text { mean (range) }\end{array}$} & \multirow{2}{*}{$\begin{array}{l}\text { Final } \\
\text { discontinuation } \\
\text { rate* }\end{array}$} \\
\hline & $\begin{array}{l}\text { Pre- } \\
\text { ULT }\end{array}$ & During ULT & $\begin{array}{l}\text { After ULT } \\
\text { discontinuation }\end{array}$ & & & \\
\hline $\begin{array}{l}\text { Gout } \\
\text { Loebl et al. }{ }^{12} \\
1974\end{array}$ & 8.4 & 5.5 & 8.8 & $36.4 \%$ (gout) & NR & $61 \%$ \\
\hline $\begin{array}{l}\text { England } \\
\text { Gast et al. }{ }^{14} \\
1986 \\
\text { The Netherlands }\end{array}$ & 9.58 & $\begin{array}{l}5.55 \\
-5.04 \text { (group } \\
\text { without relapse) } \\
-6.05 \text { (group with } \\
\text { relapse) }\end{array}$ & 9.92 & $\begin{array}{l}50 \% \text { (gout and/or } \\
\text { tophus) } \\
\text { - gout: } 50 \% \\
\text { - tophus: } 20 \%\end{array}$ & $15.8(5-29)$ & $80 \%$ \\
\hline $\begin{array}{l}\text { van Lieshout- } \\
\text { Zuidema et al. }^{13} \\
1992 \\
\text { The Netherlands }\end{array}$ & 9.58 & 5.72 & 9.41 & $\begin{array}{l}81 \% \text { (gout and/or } \\
\text { tophus) } \\
\text { - gout: } 81 \% \\
\text { - tophus: } 42.9 \%\end{array}$ & $19(4-52)$ & NR \\
\hline $\begin{array}{l}\text { Darmawan et al. } \\
2002 \\
\text { Indonesia }\end{array}$ & NR & NR & NR & $\begin{array}{l}\text { - topnus: } 42.9 \% \\
59.2 \% \text { gout }\end{array}$ & NR & $40.8 \%$ \\
\hline $\begin{array}{l}\text { Perez-Ruiz et al. }{ }^{15} \\
2011 \\
\text { Snain }\end{array}$ & 8.8 & 4.9 & 8.5 & $38.9 \%$ gout & $56(\mathrm{NR})$ & NR \\
\hline $\begin{array}{l}\text { Spain } \\
\text { Urolithiasis }\end{array}$ & & & & & & \\
\hline $\begin{array}{l}\text { Kohri et al. }{ }^{17} \\
1990\end{array}$ & NR & NR & NR & $15.1 \%$ & 9.5 & NR \\
\hline $\begin{array}{l}\text { Japan } \\
\text { Jabor }^{18} \\
1996 \\
\text { Czech Republic } \\
\text { Asymptomatic } \\
\text { hyperuricemia }\end{array}$ & NR & 5.04 & 5.58 & NR & NR & NR \\
\hline $\begin{array}{l}\text { Talaat et al. } \\
2007 \\
\text { Egypt }\end{array}$ & 9.6 & 7.5 & 12.4 & N/A & N/A & $\mathrm{N} / \mathrm{A}$ \\
\hline
\end{tabular}

ULT, urate-lowering therapy; NR, not reported; N/A, not applicable

*As of the end of the follow-up period

\section{Clinical Effect of Discontinuation in Urolithiasis}

A study in Japan by Kohri et al. included 87 patients with idiopathic calcium oxalate or calcium phosphate urinary stones. ${ }^{17}$ All were randomized to receive either allopurinol or allopurinol and trichlormethiazide. Among these, 53 discontinued ULT for at least 12 months, and were studied. Nine patients $(15.1 \%)$ had a recurrence of $\mathrm{x}$-ray-confirmed urolithiasis after allopurinol discontinuation (mean time to relapse: 9.5 months). The rate of urolithiasis relapse after ULT discontinuation was lower than that during treatment $(48.3 \%)$. The number of new stones per patient per year decreased by around $50 \%$ after ULT discontinuation compared with the treatment period (0.2-0.09 new stones/patient/year, paired $t$ test, $p<0.05)$. Urinary calcium, uric acid excretion, oxalate, and citrate increased again after discontinuation. Phosphate and magnesium levels showed no significant difference.

Jabor conducted a retrospective analysis of 24 calciumoxalate urolithiasis formers who discontinued allopurinol. ${ }^{18}$ Serum urate levels and urate output (daily output of urate, ratio of urine urate to urine creatinine, and ratio of daily urate to the patient's body mass) increased again after discontinuation of allopurinol. Urolithiasis relapse rates were not reported.
Of note, in two studies conducted in patients with gout, no patient experienced urolithiasis after ULT discontinuation. ${ }^{11,13}$

\section{Clinical Effects of Discontinuation in Asymptomatic Hyperuricemia}

Asymptomatic hyperuricemia was addressed in only one study, which included 50 patients with asymptomatic hyperuricemia and stage 3 or 4 chronic kidney disease related to hypertension. ${ }^{22}$ Patients were further classified according to their antihypertensive regimen (with or without reninangiotensin system inhibitors, RASIs). Serum urate levels, blood pressure, and kidney function were regularly measured 1 year before and 1 year after discontinuation of allopurinol. No significant changes in blood pressure were observed in patients with RASIs. Significant increases in both systolic blood pressure (124.5 \pm 7.4 to $155.6 \pm 18.4 \mathrm{mmHg}$, unpaired two-tailed Student $t$ test, $p<0.05$ ) and diastolic blood pressure $(80.5 \pm 3.2$ to $93.5 \pm 5.3 \mathrm{mmHg}, p<0.05)$ were observed 2 weeks after allopurinol discontinuation in patients with antihypertensive regimens without RASIs. In addition, kidney function declined faster in the group of patients without RASIs $(-14.1 \pm 5.3 \mathrm{ml} / \mathrm{min} /$ year, vs. $-3.9 \pm 2 \mathrm{ml} / \mathrm{min} /$ year in a group of patients treated with angiotensin-converting enzyme 


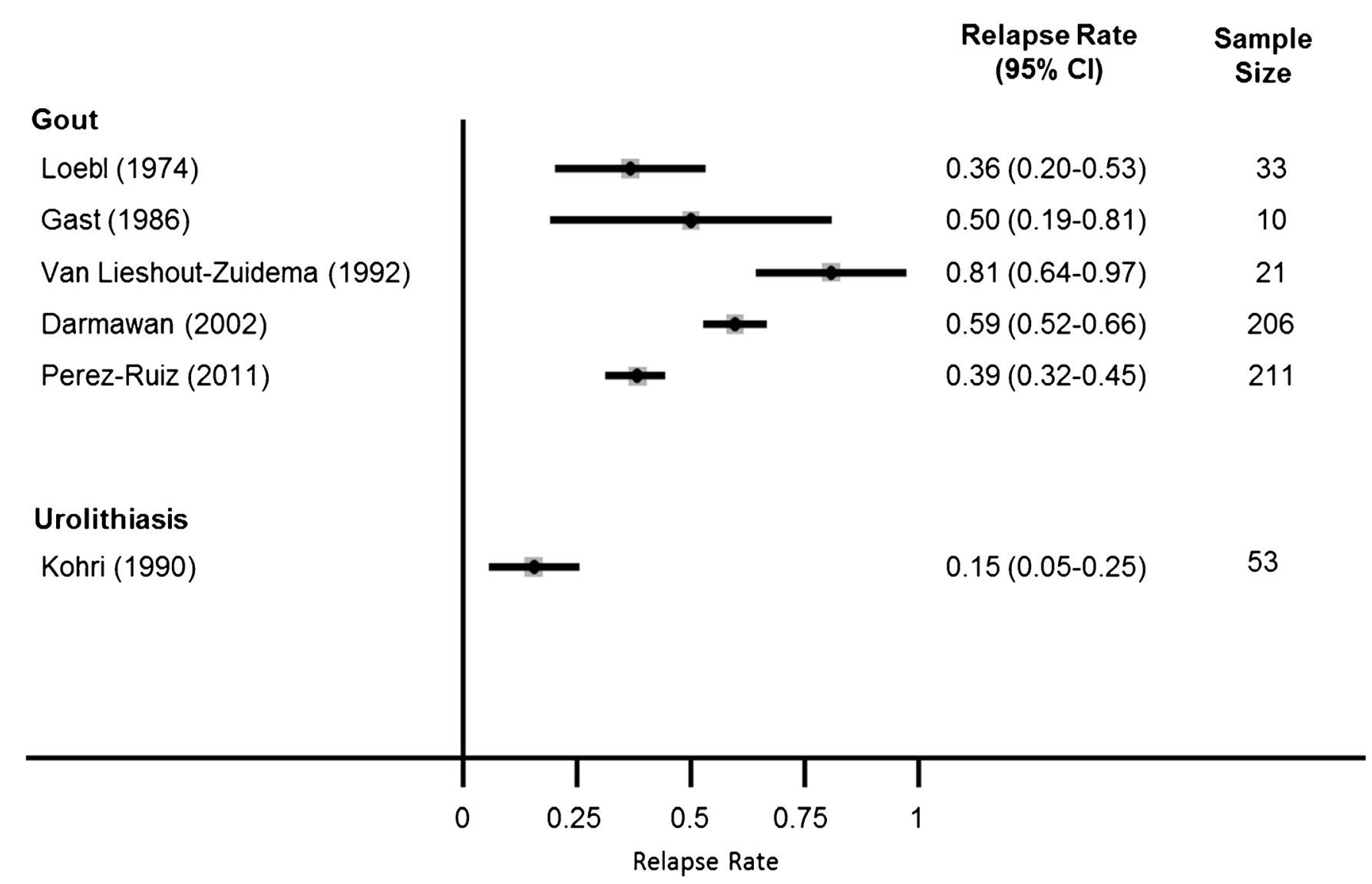

Figure 2 Relapse rates for gout and urolithiasis.

inhibitors, and $-4.1 \pm 2.2 \mathrm{ml} / \mathrm{min} /$ year in a group of patients treated with angiotensin II receptor blockers, respectively, $p<0.05)$.

\section{Additional Analyses}

In the majority of studies, serum urate levels increased again 1-3 weeks after ULT discontinuation (Table 3). Regarding kidney function, apart from the study conducted in patients with asymptomatic hyperuricemia (see above), two supplemental studies reported renal outcomes. In the study by Darmawan et al., no deterioration in renal function was recorded during the 8 years of follow-up in patients who had discontinued ULT and maintained a serum urate level $<5 \mathrm{mg} / \mathrm{dl} .{ }^{11}$ Perez et al. also reported additional data from their cohort study at the 2010 ACR meeting. ${ }^{20}$ In this analysis, 179 patients who discontinued ULT were included, with a follow-up of $34 \pm 23$ months. Renal dysfunction (Modification of Diet in Renal Disease [MDRD] renal function estimation $<60 \mathrm{ml} / \mathrm{min}$ ) increased from $13.4 \%$ of patients at baseline to $21.8 \%$ at the end of the study period. Lower renal function on ULT discontinuation, the presence of proteinuria, and the highest quartiles of serum urate levels after discontinuation were independently and significantly associated with renal dysfunction at the end of the study period.

\section{DISCUSSION}

In this work, we systematically examined the clinical and biological effects of discontinuing urate-lowering therapy, and found that the relapse rate was high in gout (36-81\% according to five studies) and was lower in urolithiasis ( $15 \%$ in one study only). These clinical effects occurred $1-4.5$ years after ULT discontinuation, and few predictive factors were identified. ULT discontinuation had no effect on creatinine levels in patients with normal renal function; however, renal function in patients with kidney disease tended to deteriorate. Clinicians should use caution in interpreting these results, given the important limitations regarding the validity of the included studies.

Serum urate levels before ULT discontinuation were consistently associated with lower gout recurrence in initial studies. ${ }^{12-14}$ However, this association was statistically confirmed only in the study by Perez-Ruiz et al., which was the largest of the studies examined. ${ }^{15}$ On this basis, Perez-Ruiz et al. suggested potency for intermittent use of ULT. ${ }^{15}$ They proposed a first period of at least 5 years of ULT maintenance targeting a serum urate level far below $6 \mathrm{mg} / \mathrm{dl}$, followed by a second period of ULT discontinuation aimed at maintaining serum urate just below the saturation level $(6-7 \mathrm{mg} / \mathrm{dl})$. An intermittent ULT regimen could thus be framed in patients discouraged with lifelong ULT treatment.

Discontinuation of ULT had no effect on creatinine serum levels in patients without renal dysfunction. In one study, 
patients with chronic kidney insufficiency (stage 3/4) and hypertension, who were not treated with RASIs, experienced a more rapid increase in creatinine serum than other patients. ${ }^{19}$ This is consistent with results observed in rat models of mild hyperuricemia, which showed an amplification of the noxious effects of angiotensin II mediated by uric acid. ${ }^{21}$ This physiopathological pathway leads to intrarenal inflammation, contributing to deterioration in kidney function. Similarly, in the largest cohort study included in this review, lower (including in patients without renal dyfunction) is associated with renal dysfunction. ${ }^{20}$ Discontinuation of ULT should thus be considered with caution in patients with kidney function impairment, especially in those with hypertension who are not treated with RASIs.

\section{Strengths and Limitations}

The strengths of this study include an exhaustive search strategy that identified a greater number of studies than a recent systematic review focusing solely on gout. ${ }^{22}$ Extending the search strategy to indications other than gout yielded consistent results (such as re-increase of serum urate level after ULT discontinuation) and brought related issues to light (such as possible blood pressure increase in patients who are not treated with RASIs).

This review is limited by the quantity and quality of the published manuscripts. The quality of the studies was low, as reflected by the MINORS scores. No randomized controlled trials were identified, and only studies with observational longitudinal designs. In such studies, the main limitation was the lack of a control group: the natural course of gout must be taken into account when maintaining ULT in order to determine the clinical effects truly associated with discontinuation of ULT. Also, a possible overlap in patients across two studies was noted. ${ }^{13,14}$ Additionally, important information was missing in some studies, in particular details about settings and time to relapse. Several studies had limited sample size: more than half of the studies included 50 or fewer patients. Such figures reduce the statistical power and the ability to provide precise estimates of relapse rates. Lastly, the study conducted in patients with asymptomatic mild hyperuricemia had the shortest follow-up (12 months); a longer follow-up would be needed to ascertain the results in this specific population, in which use of ULT is still considered off-label, albeit very common (25-91\% ULT use). ${ }^{23-25}$

The included studies also focused on more severe gout and in non-primary care settings. This makes our results difficult to translate for primary care physicians, since their patients are prone to experience less severe forms of gout, with fewer tophi and refractory gout. ${ }^{26}$ It is likely that studies assessing the effects of ULT discontinuation in primary care would report lower gout relapse rates than those identified in this review. Finally, the effects of discontinuing febuxostat have not been studied, despite its status as the second most frequently prescribed ULT, with $14.4 \%$ of new ULT users and $6 \%$ of ULT market share in the US. ${ }^{27,28}$

\section{CONCLUSION}

This systematic review suggests that relapse of gout is common, although delayed, after discontinuation of ULT. Conversely, relapses of urolithiasis were rare after ULT discontinuation. Certain factors may facilitate a framed ULT discontinuation: a sustained low serum urate level during treatment with ULT, a low serum urate level after ULT discontinuation, and the absence of kidney impairment. Further studies should consider a larger population in primary care, and discontinuation of other ULTs, including febuxostat.

\section{Acknowledgements:}

Prior Presentations: The study results have not been presented previously.

Corresponding Author: Jean-Pascal Fournier, MD, PhD; Département de médecine générale Université de Nantes, 1, rue Gaston Veil 44035, Nantes, France (e-mail:jean-pascal.fournier@univnantes.fr).

Contributors The authors thank Vera Granikov (Information Technology Primary Care Research Group, McGill University) and Chantal Maton-Elie (Bibliothèque Universitaire Santé, Université de Nantes) for their kind help in developing the search strategy. The authors also thank Sasha Mann for her help during the language editing of the manuscript.

\section{Compliance with Ethical Standards:}

Conflict of Interest: The authors declare that they have no conflict of interest.

\section{REFERENCES}

1. Briesacher BA, Andrade SE, Fouayzi H, Chan KA. Comparison of drug adherence rates among patients with seven different medical conditions. Pharmacotherapy. 2008;28(4):437-43. https://doi.org/10.1592/phco.1. 4.437 .

2. Harrold LR, Andrade SE. Medication adherence of patients with selected rheumatic conditions: a systematic review of the literature. Semin Arthritis Rheum. 2009;38(5):396-402. https://doi.org/10.1016/j. semarthrit.2008.01.011

3. De Vera MA, Marcotte G, Rai S, Galo JS, Bhole V. Medication adherence in gout: a systematic review. Arthritis Care Res. 2014;66(10):1551-9. https://doi.org/10.1002/acr.22336.

4. Solomon DH, Avorn J, Levin R, Brookhart MA. Uric acid lowering therapy: prescribing patterns in a large cohort of older adults. Ann Rheum Dis. 2008;67(5):609-13. https://doi.org/10.1136/ard.2007. 076182.

5. Zandman-Goddard G, Amital H, Shamrayevsky N, Raz R, Shalev V, Chodick G. Rates of adherence and persistence with allopurinol therapy among gout patients in Israel. Rheumatol Oxf Engl. 2013;52(6):1126-31. https://doi.org/10.1093/rheumatology/kes431.

6. Harrold LR, Mazor KM, Velten S, Ockene IS, Yood RA. Patients and providers view gout differently: a qualitative study. Chronic Illn. 2010;6(4):263-71. https://doi.org/10.1177/1742395310378761.

7. Singh JA. Facilitators and barriers to adherence to urate-lowering therapy in African-Americans with gout: a qualitative study. Arthritis Res Ther. 2014;16(2):R82. https://doi.org/10.1186/ar4524.

8. Chandratre P, Mallen CD, Roddy E, Liddle J, Richardson J. "You want to get on with the rest of your life": a qualitative study of health-related quality of life in gout. Clin Rheumatol. 2016;35(5):1197-1205. https:// doi.org/10.1007/s10067-015-3039-2.

9. Spaetgens B, Pustjens T, Scheepers LEJM, Janssens HJEM, van der Linden S, Boonen A. Knowledge, illness perceptions and stated clinical practice behaviour in management of gout: a mixed methods study in general practice. Clin Rheumatol. 2016;35(8):2053-2061. https://doi. org/10.1007/s10067-016-3212-2. 
10. Slim K, Nini E, Forestier D, Kwiatkowski F, Panis Y, Chipponi J. Methodological index for non-randomized studies (MINORS): development and validation of a new instrument. ANZ J Surg. 2003;73(9):712-6.

11. Darmawan J, Rasker JJ, Nuralim H. The effect of control and selfmedication of chronic gout in a developing country. Outcome after 10 years. J Rheumatol. 2003;30(11):2437-43.

12. Loebl WY, Scott JT. Withdrawal of allopurinol in patients with gout. Ann Rheum Dis. 1974;33(4):304-7. https://doi.org/10.1136/ard.33.4.304.

13. van Lieshout-Zuidema MF, Breedveld FC. Withdrawal of longterm antihyperuricemic therapy in tophaceous gout. J Rheumatol 1993;20(8): 1383-5.

14. Gast LF. Withdrawal of longterm antihyperuricemic therapy in tophaceous gout. Clin Rheumatol 1987;6(1):70-3. https://doi.org/10. $1007 / \mathrm{BF} 02201004$

15. Perez-Ruiz F, Herrero-Beites AM, Carmona L. A two-stage approach to the treatment of hyperuricemia in gout: the "dirty dish" hypothesis. Arthritis Rheum 2011;63(12):4002-6. https://doi.org/10.1002/art. 30649.

16. Wallace SL, Robinson H, Masi AT, Decker JL, McCarty DJ, Yü TF. Preliminary criteria for the classification of the acute arthritis of primary gout. Arthritis Rheum 1977;20(3):895-900.

17. Kohri K, Kodama M, Katayama Y, et al. Allopurinol and thiazide effects on new urinary stone formed after discontinued therapy in patients with urinary stones. Urology 1990;36(4):309-14.

18. Jabor A. Kidney stone clinic: ten years of experience. Ned Tijdschr Klin Chem 1996;21(1):8-10.

19. Talaat KM, el-Sheikh AR. The effect of mild hyperuricemia on urinary transforming growth factor beta and the progression of chronic kidney disease. Am J Nephrol 2007;27(5):435-40. https://doi.org/10.1159/ 000105142

20. Perez-Ruiz F, Herrero-Beites AM, Miguel A. Serum urate levels have an impact on renal function in patients with gout withdrawing uratelowering therapy (ULT). Arthritis Rheum 2010;62(Suppl 10):159. https://doi.org/10.1002/art.30649.
21. Mazzali M, Hughes J, Kim YG, et al. Elevated uric acid increases blood pressure in the rat by a novel crystal-independent mechanism. Hypertension 2001;38(5):1101-6.

22. Shekelle PG, FitzGerald J, Newberry SJ, et al. Management of Gout. Rockville: Agency for Healthcare Research and Quality (US); 2016. http://www.ncbi.nlm.nih.gov/books/NBK356141/. Accessed February $16,2017$.

23. Mikuls TR, Farrar JT, Bilker WB, Fernandes S, Saag KG. Suboptimal physician adherence to quality indicators for the management of gout and asymptomatic hyperuricaemia: results from the UK General Practice Research Database (GPRD). Rheumatol Oxf Engl 2005;44(8): 1038-1042. https://doi.org/10.1093/rheumatology/keh679.

24. Pasina L, Brucato AL, Djade CD, et al. Inappropriate prescription of allopurinol and febuxostat and risk of adverse events in the elderly: results from the REPOSI registry. Eur J Clin Pharmacol 2014;70(12):1495-1503. https://doi.org/10.1007/s00228-014-1752-4.

25. Banse C, Fardellone P, Paccou J. Prevalence of treatment of hyperuricemic in patients admitted to the Rheumatology ward and evaluation of compliance with the 2012 ACR Guidelines. Joint Bone Spine Rev Rhum 2014;81(5):461-2. https://doi.org/10.1016/j.jbspin. 2014.02.012.

26. Singh JA, Sarkin A, Shieh M, et al. Health Care Utilization in Patients with Gout. Semin Arthritis Rheum 2011;40(6):501-11. https://doi.org/ 10.1016/j.semarthrit.2010.07.001.

27. Smolen LJ, Gahn JC, Mitri G, Shiozawa A. The Budget Impact of Increased Use of Febuxostat in the Management of Gout: A US Health Plan Managed Care Pharmacy and Medical Costs Perspective. Clin Ther 2016;38(7):1710-25. https://doi.org/10.1016/j.clinthera.2016.05.007.

28. Kim SC, Schmidt BMW, Franklin JM, Liu J, Solomon DH, Schneeweiss S. Clinical and health care use characteristics of patients newly prescribed allopurinol, febuxostat and colchicine for gout. Arthritis Care Res 2013;65(12):2008-14. https://doi.org/10.1002/acr.22067. 